Matière Condensée de Paris have tackled both problems by using a simple aerosolbased process to create extremely effective aluminosilicate catalysts.

By spray-drying a solution of precursors, template and structure-directing agents, amorphous spherical particles with large pores were formed in a few seconds. The drying process gradually concentrates the species until micelles form at the same time as the inorganic precursors react. This technique removes the need for zeolite 'seeds' in preformation steps or acidification steps involving sodium, and the product is simply collected from a filter.

The catalysts obtained through this process were three times more active in $m$-xylene isomerization than industrially used reference samples. Furthermore, this high activity was maintained for longer, because the new materials were less deactivated by coke formation. Sanchez and colleagues prepared samples with a range of pore size, aluminium content and acid sites of different strengths, but found that the microporous surface was the parameter that determined the catalytic activity.

\section{ICE NUCLEATION}

\section{Pentagonal patterns}

Nature Mater. 8, 427-431 (2009)

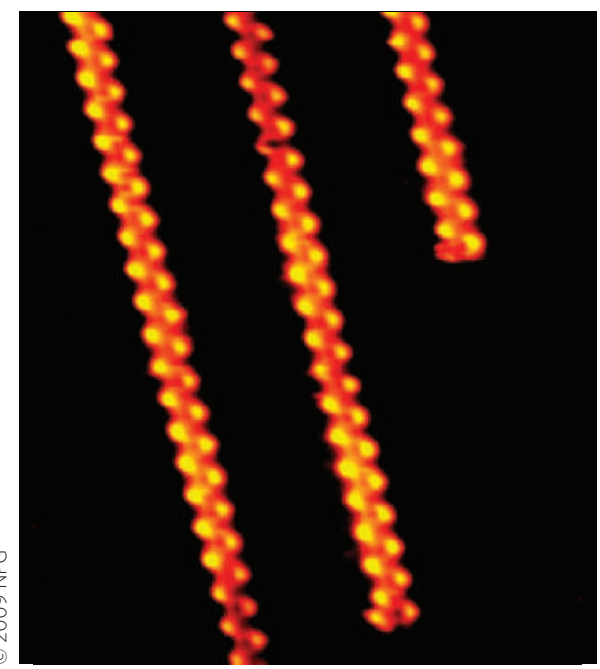

The hexagonal crystal structure of ice is well known in the bulk phase - but the initial steps of ice formation on a surface, despite its relevance in fields ranging from biology to atmospheric chemistry, remain unclear. Numerous studies have been carried out on this nucleation process and, although they are not always in agreement about the structure of the ice-water films formed, they have all so far suggested hexagonal arrangements of the water molecules.
Angelos Michaelides and Javier Carrasco at the London Centre for Nanotechnology and colleagues at the University of Liverpool have now revealed a structure based on pentagonal patterns. They used scanning tunnelling microscopy to study the nucleation of ice on a copper(110) substrate, a striped surface with alternate ridges and trenches. The images clearly showed the formation of regular one-dimensional chains along the ridges. Combining reflection absorption infrared spectroscopy and density functional theory calculations, the researchers found that these chains were made of pentagonal units lying flat on top of the ridges.

The pentagonal arrangement maximizes interactions with the metal surface while minimizing the strain within the overall layer. Future studies are now likely to take into account the balance of water-metal and water-water interactions, rather than focus on the formation of hexagonal structures.

\section{MICROFLUIDIC DRUG DETECTION}

\section{Know when to fold}

\section{J. Am. Chem. Soc. 131, 4262-4266 (2009)}

A range of techniques can be used to detect analytes in complex aqueous solutions, making use of the changes in properties such as charge or mass that occur when the analyte binds to the sensor. Such adsorption-based devices suffer from an inability to differentiate between genuine target molecules and contaminants giving false positives when assessing unprocessed samples.

Sensors that use analyte-induced changes in biomolecule conformation do not have this problem because the nonspecific binding of non-target molecules does not induce the conformational changes required to create a signal. Now, Tom Soh and colleagues at the University of California, Santa Barbara, have developed such a sensor, using the conformational changes of a DNA aptamer to sense cocaine in blood serum.

The device consists of a microfluidic chamber that contains a reference electrode and several gold electrodes functionalized with a layer of 32-base DNA aptamers. Importantly, these have a redox-active methylene blue moiety attached to their free end, and they remain unfolded until the specific binding of cocaine. The targetinduced folding brings the methylene blue closer to the gold electrode, increasing the efficiency of electron transfer from methylene blue to the electrode and in turn increasing the measurable current. Changes in current can thus be related to cocaine concentration.

\section{blogroll $^{\text {( }}$}

\section{I'd like to thank...}

The etiquette of acknowledgements and the importance of stupidity are discussed and dissected.

The tricky subject of acknowledgements in journal articles is raised by

FemaleScienceProfessor (http://tinyurl. com/cfuq8v). She outlines some thorny problems to consider when drafting those brief final words that appear at the end of practically every paper. Did anyone influence the research, but not contribute enough to be a full co-author? Should you thank anonymous reviewers? Should you try to gratuitously shoe-horn in 'a bunch of famous people who are aware l exist'? And what if you thank people who don't want to be mentioned? Aside from Mark $P$ who wanted to thank the Lindt chocolate company for "greatly enhancing his graduate work", most people commenting didn't think thanking anonymous reviewers was particularly useful - and most of them were commenting anonymously too! Although a few people said they weren't bothered by being thanked when they didn't feel they really deserved it, the crucial point was raised that this could be done to avoid them being used as a reviewer - sneaky.

At The Chemistry Blog (http://tinyurl. $\mathrm{com} / \mathrm{d} x w w d w)$, Boyie picks up on an essay 'The Importance of Stupidity in Scientific Research' published in Journal of Cell Science (121, 1771; 2008). Apart from allowing people to breathe a secret sigh of relief, if you don't feel stupid occasionally you're not picking the right research problems, you're not working hard enough to get stupefying results or you don't really understand enough! So unless you feel stupid, you're being stupid in another way. The Chemist (from blog My Chemical Journey, http://mychemicaljourney.blogspot. com) reminded readers of James Watson "saying that breakthroughs are sometimes owed to 'the principle sloppiness [sic]' to get non-reproducible results". So, if you're feeling sloppy and stupid today, phone the Nobel Prize Committee: your big breakthrough might be round the corner! 